\title{
Atypical clinical presentations in right hemispheric infarcts: complex stories beyond neurological examination and diffusion-weighted magnetic resonance imaging
}

\author{
Jaeho Kim, Jaehong Park, Hyun Hee Kang, Oh Young Bang \\ Departments of Neurology, Samsung Medical Center, Sungkyunkwan University School of Medicine, Seoul, Korea
}

Received: July 17, 2018

Revised: August 23, 2018

Accepted: August 28, 2018

Corresponding author:

Oh Young Bang

Department of Neurology,

Samsung Medical Center,

Sungkyunkwan University

School of Medicine, 81 Irwon-ro,

Gangnam-gu, Seoul 06351, Korea

Tel: +82-2-3410-3599

E-mail:

ohyoung.bang@samsung.com

\section{ABSTRACT}

Although, both neurological examination and neuroimaging represent the main methods for the anatomical localization of lesions in stroke patients, atypical anatomical-functional representation of cortical lateralized syndromes can occur. Herein, we introduce three cases of atypical representation of left hemispheric syndromes with right hemispheric lesions and we explore the possible mechanisms underlying such disorders and their clinical implications.

Keywords: Cerebral infarction; Crossed aphasia; Magnetic resonance imaging

\section{INTRODUCTION}

Both neurological examination and neuroimaging, including brain magnetic resonance imaging (MRI), are the main methods for the anatomical localization of lesions in stroke patients. Considering that the language network is found in the perisylvian region of the dominant (generally left) hemisphere, lesions localized in this area result in aphasia. Additionally, lesions in the left inferior parietal lobule can produce various disorders, including agraphia, acalculia, left-right disorientation, finger agnosia (Gerstmann syndrome), and ideomotor apraxia. In contrast, right-sided lesions (non-dominant hemisphere) can cause hemineglect of the left hemibody or extrapersonal space. Neurological examinations represent a particularly important tool for treating patients with acute ischemic stroke as, in most cases, the revascularization therapy procedure (either intravenous thrombolysis using tissue plasminogen activator or endovascular therapy using stentriever) is chosen with the aid of neurological examination and computed tomographic (CT) findings.

It is established that right hemispheric lesions rarely cause aphasia in patients (right- or distributed under the terms of the Creative Commons Attribution Non-Commercial License (http:// creativecommons.org/licenses/ by-nc/4.0/). left-handed) who have their language representation areas in the right hemisphere [1]. However, when this disorder occurs in right-handed patients, the term 'crossed aphasia' is used [2,3]. Besides language function disorders, atypical anatomical-functional correlations of the repre- 
sentation of other lateralized syndromes can occur. In this case report study, we introduce three cases of atypical representation of left hemispheric syndromes following right hemispheric lesions.

\section{CASE REPORTS}

Case 1. Transcortical mixed crossed aphasia and neglect syndrome without hemiplegia

A 66-year-old right-handed man suddenly developed aphasia. He was taking an antiplatelet agent for stable angina and other medications for treating hypertension and diabetes. He was a heavy smoker and alcoholic. Upon neurologic examination, both his strength and sensation perception were normal. In contrast, in the speech evaluation, his fluency, comprehension, and naming functions were decreased, except for repetition, suggesting transcortical mixed aphasia pattern. Left side neglect, including motor, tactile, and auditory extinction, was noted whereas an absence of abnormality was observed in apraxia tests. Brain MRI revealed acute infarcts in the right middle cerebral artery (MCA) border zone (Fig. 1A). Specifically, the perfusion-weighted MRI showed a perfusion delay associated to the lesion (Fig. 1B) whereas the magnetic resonance (MR) angiography identified both a severe narrowing and stenosis in the right internal carotid artery (Fig. 1C, D), suggesting the etiology of his stroke to be the large artery atherosclerosis. Additionally, polyvascular CT angiography revealed both a severe stenosis with soft plaques in the coronary arteries and an occlusion of the right common iliac and the right external and internal iliac arteries. Given the man's large artery atherosclerosis, he was treated by adding antiplatelet to dual antiplatelet and high dose statin medication.
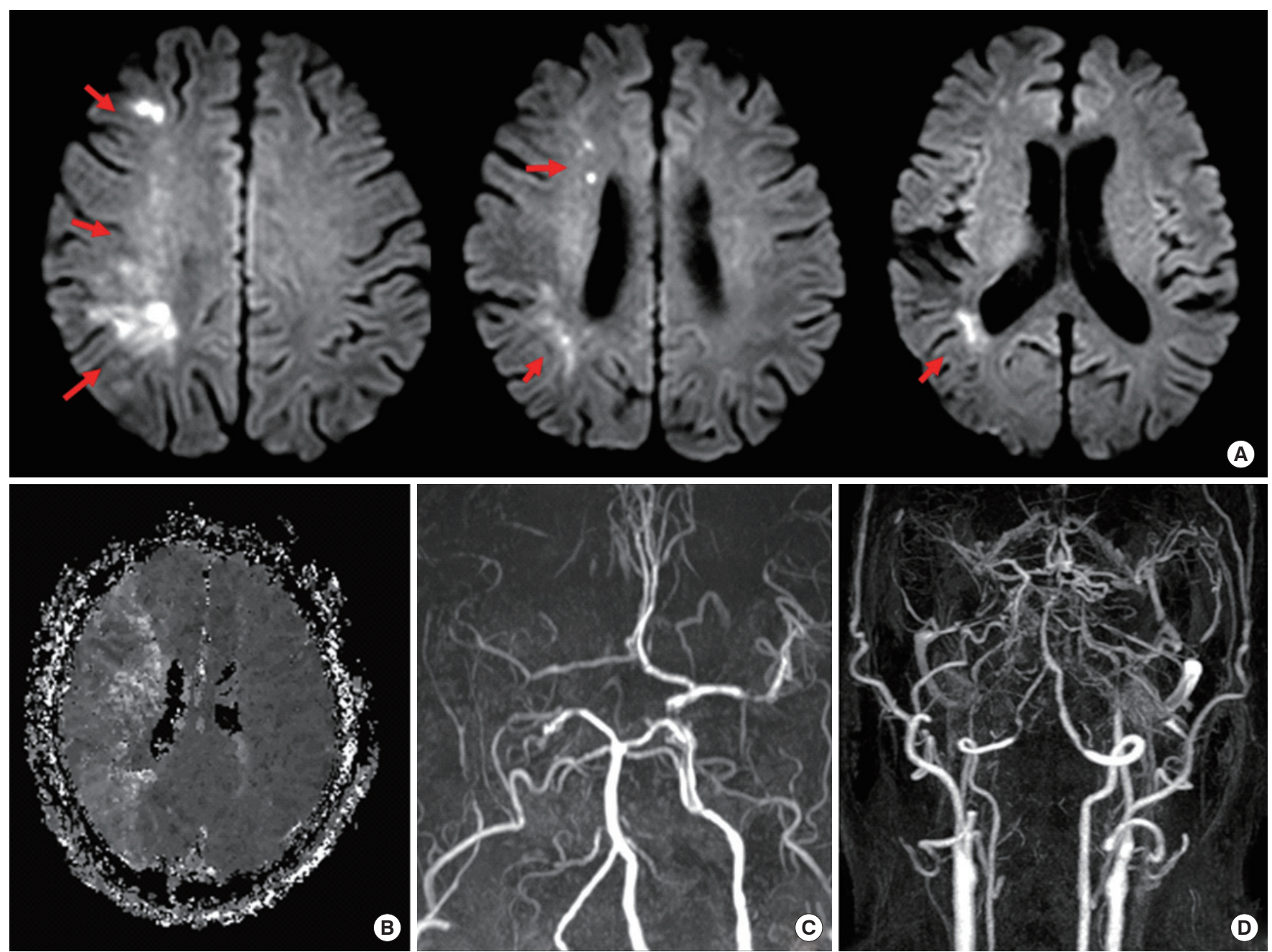

Fig. 1. Case 1. (A) A diffusion-weighted image showing acute cerebral infarcts in the right middle cerebral artery (MCA) border zone (arrows). (B) A magnetic resonance (MR) perfusion image showing perfusion delay in the right MCA territory. (C, D) A MR angiography showing a severe narrowing in the right internal carotid artery and an almost total occlusion in the petrous segment. 
A follow-up study was conducted 5 days following the syndrome onset and tests for language function and neglect syndrome showed an almost complete recovery.

\section{Case 2. Transcortical sensory aphasia, ideomotor} apraxia, and Gerstmann syndrome without neglect syndrome

A 70-year-old left-handed man developed speech disturbance and lost his ability of using his tools following a right temporoparietal infarction. He was not taking oral anticoagulants, although he was diagnosed with arrhythmia on routine checkup. His problem started with an observed difficulty in using the tools which included a scissor and knife handling, as it was noticed by his wife. The neuropsychological examinations revealed transcortical sensory aphasia (im- paired speech comprehension, however, repetition and fluency of speech were intact), left-sided weakness and ideomotor apraxia of learned behavior. After recovery of his language function, he reported finger agnosia, right-left confusion, agraphia and acalculia, indicative of the Gerstmann syndrome. Because visual and sensory involvement was identified, extinction of these two sensory abilities could not be tested. However, anosognosia, asomatognosia, and anosodiaphoria were not observed. As the brain MRI showed both multiple acute infarcts in the right posterior parietal cortex and normal brain vessels, a cardioembolic embolism was suggested (Fig. 2A, B). Functional MRI revealed the right hemispheric dominancy on language function (Fig. 2C). Both his language dysfunction and Gerstmann syndrome disappeared within a few months after the symptoms onset.
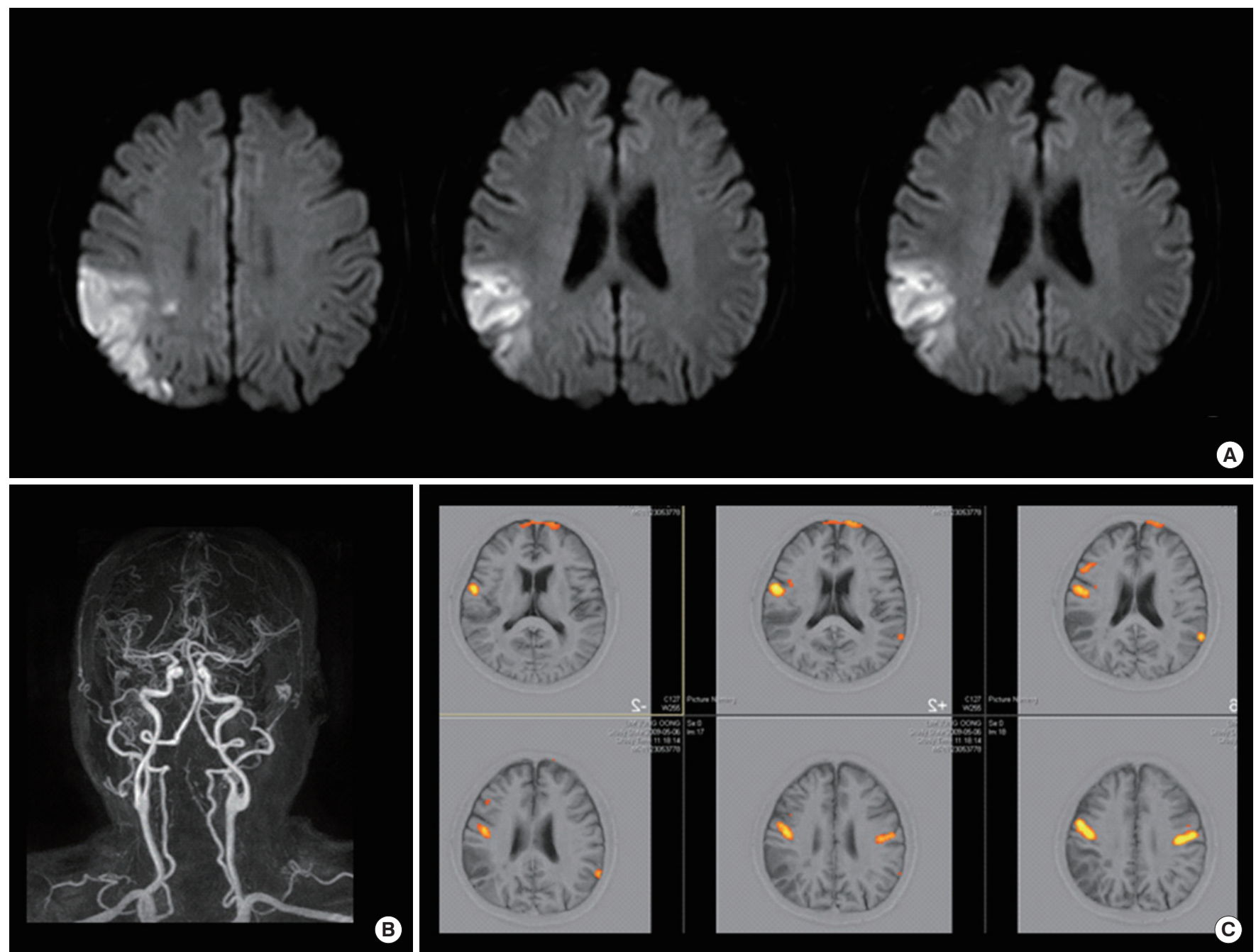

Fig. 2. Case 2. (A) A diffusion-weighted image showing acute cerebral infarcts in the right parietal cortex. (B) Normal magnetic resonance angiography findings. (C) A functional magnetic resonance image showing the right hemispheric dominancy of this patient's language function. Speech generated Blood Oxygen Level Dependent (BOLD) more signal changes in the right hemisphere. 

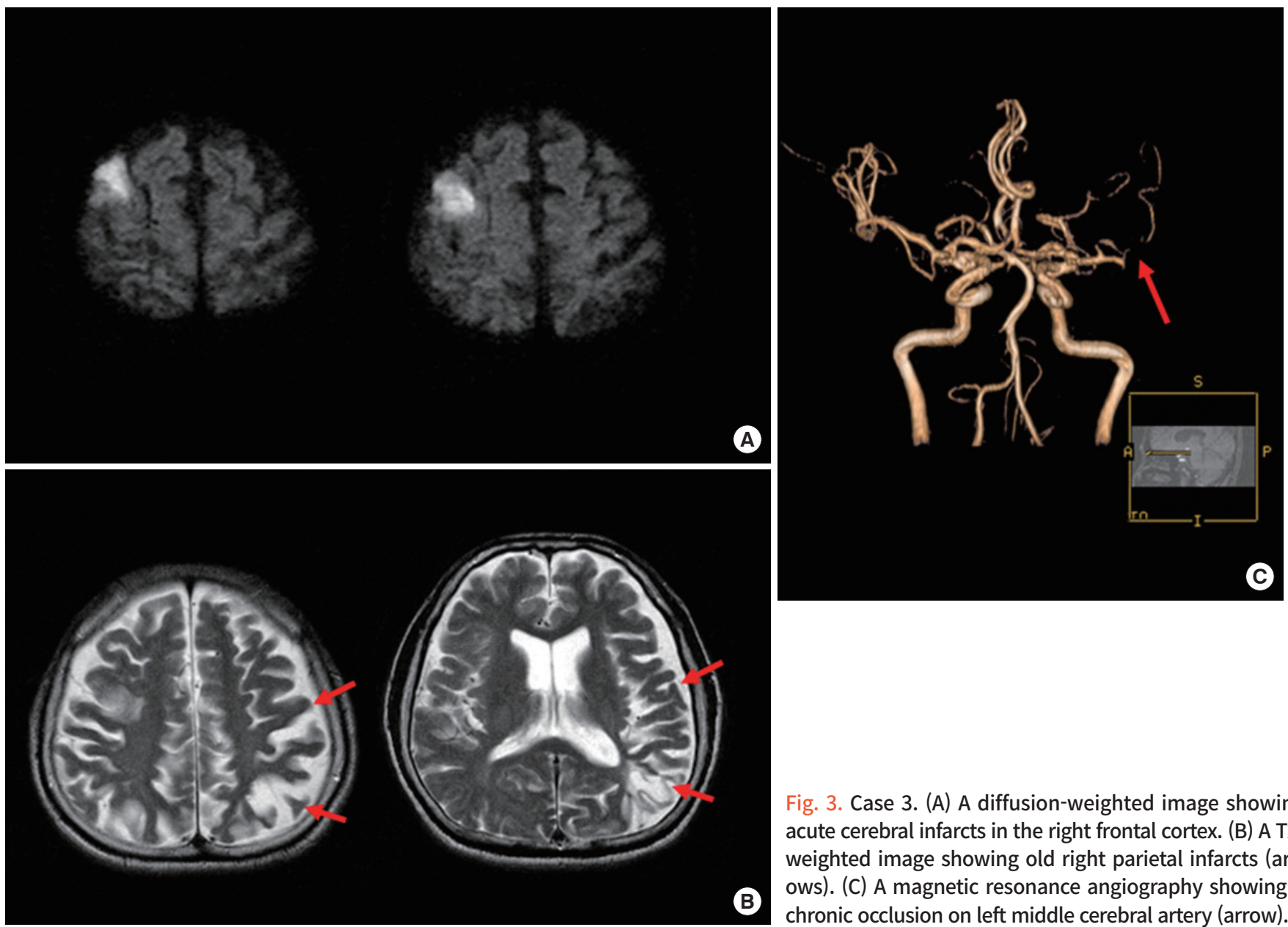

Fig. 3. Case 3. (A) A diffusion-weighted image showing acute cerebral infarcts in the right frontal cortex. (B) A T2weighted image showing old right parietal infarcts (arrows). (C) A magnetic resonance angiography showing a chronic occlusion on left middle cerebral artery (arrow).

Case 3. Transcortical aphasia and ideomotor apraxia without neglect syndrome and hemiplegia

A 71-year-old right-handed man visited the emergency center due to some speech difficulties and unusual behavior. He reported that, while working as a guard, he tried to open a door with a key but he did not know how to use it and that he tried to make a call but he did not know which button to press. He also stated that he developed speech problem that same day. Fourteen years before, he experienced a right hemiplegia and speech disturbance; however, he recovered without complications. He was a current smoker but did not have vascular risk factor, such as hypertension and diabetes. During his neurological examinations, although he was alert and had a fluent speech, he could not follow verbal commands. He could not imitate gestures, such as brushing and combing. His neuropsychological examinations revealed both transcortical sensory aphasia and ideomotor apraxia. The rest of his neurological examinations, which included motor and sensory function was normal. Hemispatial neglect syndrome was not observed. Brain MRI showed acute infarct on the right frontal cortex (Fig. 3A). Specifically, the T2-weighted image revealed old infarcts on the left parietal cortex and the MR angiography identified a chronic occlusion of the left middle cerebral artery (Fig. 3B, C). His language function and apraxia disappeared within a few weeks after the symptoms onset.

\section{DISCUSSION}

Although a combination of right and left hemispheric syndromes was previously reported in one ambidextrous patient with a right hemispheric hemorrhage [4], the cases here presented revealed that the combination of dominant (aphasia, ideomotor apraxia, and Gerstmann syndrome) and nondominant (hemispatial neglect) hemispheric syndromes can occur in patients with right hemispheric lesions, either when right- or left-handed. In addition, although there is evidence of aphasia in patients with a right hemisphere language dominance [1], this is unlikely to accompany the Gerstmann syndrome or the apraxia associated to the right hemisphere lesion. We report patients who developed full acute neuro- 
psychological symptoms in the dominant hemisphere after right hemispheric infarction. To the best of our knowledge, this is the first evidence of both neglect syndrome without hemiplegia (case 1) and the Gerstmann syndrome without the hemineglect syndrome (case 2).

The cases here described highlighted that the complexity of the brain functions depended on the involved brain areas (case 1), the handedness of the patients (case 2), and their brain plasticity (case 3). The possible mechanisms underlying the atypical representation of left hemispheric syndromes with right hemispheric lesions are the following. In the first case, the patient was right-handed and showed crossed aphasia, a rare form of aphasia which was estimated in a recent study to represent only $0.4 \%$ to $2.0 \%$ of all aphasia cases [2]. Cortical border zone infarction is reportedly associated with mixed transcortical aphasia and showed rapid recovery and general good prognosis [5]. Typically, the neglect syndrome is accompanied by hemiplegia due to extensive lesions of right hemisphere. A possible explanation of the observed hemineglect syndrome without hemiplegia could be the impaired connection between the white matter within the right hemisphere due to the cortical border zone infarcts, without involving the corticospinal tract. The second case described a left-handed man, with a right hemispheric dominancy of his language function (as seen through a functional MRI), suggesting the possibility in humans of a bihemispheric representation of the left hemispheric functions (both language function and Gerstmann syndrome) in left handers. A functional MRI study showed differential functional activity patterns and an effective connectivity among brain regions between right- and left-handed patients [6]. Specifically, a better bihemispheric coordination and an increased interhemispheric communication was seen in left-handed patients [6]. Lastly, neuroplasticity may represent a possible explanation of the atypical representation of cortical function observed in our third case. In fact, as the brain has the capacity to generate new neurons (neurogenesis), and rewire its connections (neuroplasticity), the surviving cortical regions are able to assume the same functions as the ones present in the stroke-damaged areas. Ago et al. [7] identified a patient who developed a small infarct in the left hemisphere which resulted in left hemiplegia. In addition, this patients had a history of right putaminal hemorrhage and a functional MRI study showed cortical reorganization of his left motor function on the ipsilateral left hemisphere. Similarly, our case 3 had a history of both large left hemispheric infarct and left MCA occlusion, therefore cortical re- organization of his left hemispheric function into his right hemisphere may have happened. In fact, previous infarct and a long-term hemodynamic impairment of the left hemisphere could have induced cerebral reorganization prior to the stroke onset [8].

The clinical implications that may result from the cases described in this report are as followings. To start, neurological examinations may denote some limitations in the assessment of patients with acute ischemic stroke, especially in those with right hemispheric lesions. In these instances, a more careful evaluation, which may include reporting both the patients' history of previous strokes and handedness, may be beneficial. In addition, although initial stroke severity usually correlates to long-term functional outcome, such a correlation may not be observed in patients with right hemispheric lesions and atypical representation. Some common features of our patients were transcortical-type aphasia and rapidly improving hemispheric syndromes, suggesting a favorable outcomes even without revascularization therapy. Further studies on the use of functional neuroimaging, including functional MRI and diffusion tensor image, are needed to investigate the precise mechanisms of atypical clinical presentation of right hemispheric infarcts.

\section{CONFLICTS OF INTEREST}

No potential conflict of interest relevant to this article was reported.

\section{ACKNOWLEDGMENTS}

This study was supported by grants from the National Research Foundation of Korea (NRF) funded by Ministry of Science, ICT \& Future Planning (NRF-2017R1C1B2002624 and NRF-2014R1A1A1004645).

\section{ORCID}

Oh Young Bang https://orcid.org/0000-0002-7962-8751

\section{REFERENCES}

1. Dewarrat GM, Annoni JM, Fornari E, Carota A, Bogousslavsky J, Maeder P. Acute aphasia after right hemisphere stroke. J Neurol 2009;256:1461-7.

2. Kim HS, Shin JB, Kim JM. Crossed transcortical motor aphasia, left spatial neglect, and limb and magnetic aprax- 
ia due to right anterior cerebral artery infarction. Ann Rehabil Med 2011;35:949-53.

3. Coppens P, Hungerford S, Yamaguchi S, Yamadori A. Crossed aphasia: an analysis of the symptoms, their frequency, and a comparison with left-hemisphere aphasia symptomatology. Brain Lang 2002;83:425-63.

4. Dozono K, Hachisuka K, Ohnishi A, Ogata H. Gerstmann's syndrome and ideational apraxia with a right cerebral hemispheric lesion: a case report. Neurocase 1997;3:616.

5. Flamand-Roze C, Cauquil-Michon C, Roze E, Souillard-Scemama R, Maintigneux L, Ducreux D, et al. Aphasia in border-zone infarcts has a specific initial pattern and good long-term prognosis. Eur J Neurol 2011;18:1397-401.

6. Gao Q, Wang J, Yu C, Chen H. Effect of handedness on brain activity patterns and effective connectivity network during the semantic task of Chinese characters. Sci Rep 2015;5:18262.

7. Ago T, Kitazono T, Ooboshi H, Takada J, Yoshiura T, Mihara $\mathrm{F}$, et al. Deterioration of pre-existing hemiparesis brought about by subsequent ipsilateral lacunar infarction. J Neurol Neurosurg Psychiatry 2003;74:1152-3.

8. Cauquil-Michon C, Flamand-Roze C, Denier C. Borderzone strokes and transcortical aphasia. Curr Neurol Neurosci Rep 2011;11:570-7. 